\title{
Intra-Vascular Occlusion of the Aorta for Massive Pelvic Trauma: A New Application
}

\author{
Paul Hanna ${ }^{\mathrm{a}, \mathrm{c}}$, Paul Seo ${ }^{\mathrm{b}}$, John Yoon ${ }^{\mathrm{a}}$, Manrique Guerrero ${ }^{\mathrm{a}}$, Hoan Bui ${ }^{\mathrm{a}}$, \\ Robert Madlinger ${ }^{\mathrm{a}}$, Jamshed Zuberi ${ }^{\mathrm{a}}$
}

\begin{abstract}
Globally, trauma remains the leading cause of morbidity and mortality for all age groups with uncontrolled hemorrhage as the most common form of preventable death in the trauma setting. Specifically, non-compressible torso hemorrhage in trauma patients is known to have high mortality rates. Open aortic cross clamping via anterolateral thoracotomy has been the standard approach, but the procedure carries a high mortality risk. Resuscitative endovascular balloon occlusion of the aorta (REBOA) is a technique that promptly controls hemorrhage, increases cardiac afterload, and increases central aortic pressure. REBOA in different forms has existed since the Korean War in 1950. It involves the insertion of a balloon into the aorta via a femoral access approach that is subsequently inflated to provide hemostasis. Despite the potential of REBOA to save lives, many non-vascular surgeons and emergency healthcare providers may be reluctant to use the new procedure. This may be due to a lack of knowledge, skill, or equipment required to perform the procedure. We present a case of a patient with multiple pelvic injuries and massive bleeding for which REBOA was utilized safely and correctly.
\end{abstract}

Keywords: Trauma; Resuscitative endovascular balloon occlusion of the aorta; Aortic occlusion; Hemorrhagic shock; Hemorrhage; Pelvic bleeding; Pelvic hemorrhage

\section{Introduction}

Non-compressible torso hemorrhage in trauma patients is known to have high mortality rates. Open aortic cross clamping via anterolateral thoracotomy has been the standard approach, but the procedure carries a high mortality risk [1]. Recently a minimally invasive endovascular approach has been developed to occlude the aorta proximally and control torso

Manuscript submitted May 7, 2018, accepted May 16, 2018

aSaint Joseph's Regional Medical Center, Paterson, NJ 07503, USA

${ }^{b}$ New York Medical College, Valhalla, NY 10595, USA

'Corresponding Author: Paul Hanna, Department of Surgery, Saint Joseph's

Regional Medical Center, 703 Main Street, Paterson, NJ 07503, USA.

Email: PaulHannaDO@gmail.com

doi: https://doi.org/10.14740/jcs349w hemorrhage.

Resuscitative endovascular balloon occlusion of the aorta (REBOA) is a technique that promptly controls hemorrhage, increases cardiac afterload, and increases central aortic pressure. Studies demonstrate that REBOA surpasses the aortic cross-clamping procedure with higher survival rates and fewer premature deaths [1, 2]. REBOA can be used as a proactive measure when a risk of cardiovascular collapse is identified.

Despite the potential of REBOA to save lives, many nonvascular surgeons and emergency healthcare providers may be reluctant to use the new procedure. This may be due to a lack of knowledge, skill, or equipment required to perform the procedure. However, recent studies demonstrate that with proper training, nonsurgical providers can properly place REBOA catheters effectively in austere prehospital settings [3].

REBOA is a novel procedure, with not many documented successful cases for pelvic trauma. In this paper we present a case of a patient with multiple pelvic injuries and massive bleeding for which REBOA was utilized safely. This case is unique in that there is sound coordination between trauma surgery, critical care, vascular surgery, orthopedic surgery and urology in order to effectively manage the patient.

\section{Case Report}

A 23-year-old male with no prior medical history was brought into the emergency department following a 10 -foot fall. The patient stated he was climbing a wall, fell backwards, and subsequently a large concrete block fell onto his pelvis. He complained of pain in the pelvic region and was unable to move his lower extremities secondary to the pain. He denied hitting his head or losing consciousness, and was able to recollect the events.

His vitals in the trauma bay were temperature $35.7^{\circ} \mathrm{C}$, pulse 58 beats/min, blood pressure $112 / 63 \mathrm{~mm} \mathrm{Hg}$, respiratory rate 14 breaths/min with saturations at $100 \%$ on $3 \mathrm{~L}$ of oxygen nasal cannula. Routine labs and an arterial blood gas were performed: hemoglobin $11.8 \mathrm{mg} / \mathrm{dL}$, hematocrit $36 \%$, INR 1.1, $\mathrm{pH} 7.41$, lactate $1.4 \mathrm{mg} / \mathrm{dL}$, base deficit -2.1 .

On initial assessment, the patient's airway was intact with normal cardiopulmonary, neurological and abdominal exams. On musculoskeletal exam the patient had tenderness with palpation of the pelvis. Vascular exam confirmed palpable femoral and dorsalis pedis pulses bilaterally.

A fast exam was performed which was negative in all four 


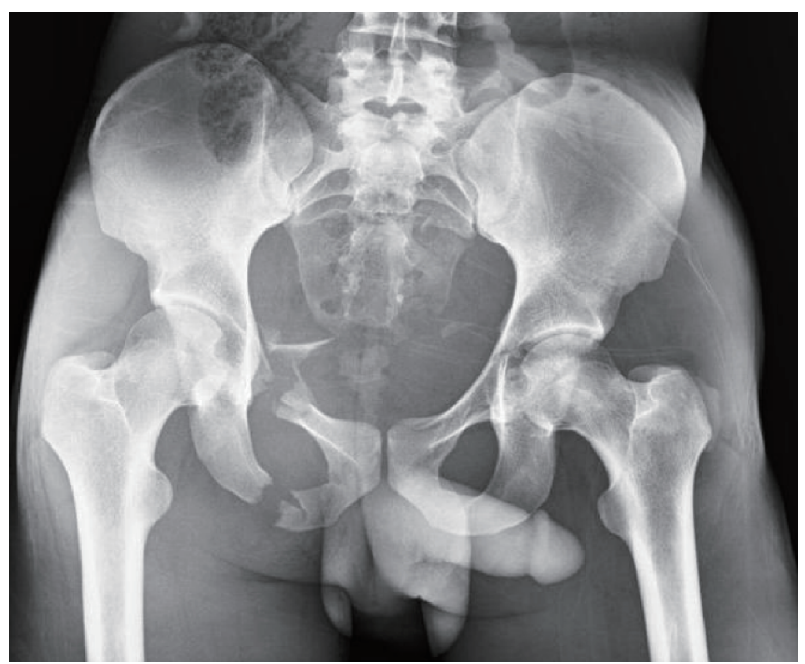

Figure 1. Pelvic X-ray showing fractures of the right superior and inferior rami, left superior ramus, and left acetabulum.

regions. A bedside chest X-ray showed clear lung fields bilaterally. Pelvic X-ray showed a comminuted, displaced fracture of the right superior and inferior pubic rami as well as nondisplaced fractures of the left superior pubic ramus and left acetabulum (Fig. 1).

En route to the CT scan, the patient became hypotensive to $70 / 50 \mathrm{~mm} \mathrm{Hg}$. Two units of packed red blood cells (pRBCs) were immediately transfused with an initial response to 100 - $110 \mathrm{~mm} \mathrm{Hg}$ systolic BP. However, he became hypotensive again to $80 \mathrm{~mm} \mathrm{Hg}$ systolic. The patient was then transferred back to the trauma bay with activation of massive transfusion protocol (MTP).

In the trauma bay, a pelvic binder was placed and it was decided to deploy a REBOA catheter. The left common femoral artery was accessed endovascularly under ultrasound guidance with an $18 \mathrm{G}$ needle. The REBOA catheter was inserted with a 7F sheath via the Seldinger technique. The catheter was measured from the xyphoid to the femoral artery cannulation site, which was approximately $30 \mathrm{~cm}$ for balloon placement in the infrarenal aorta, zone III. An abdominal X-ray was performed to confirm placement (Fig. 2). The balloon was then inflated using $5 \mathrm{ml}$ of saline and the systolic blood pressure increased to $110 \mathrm{~mm} \mathrm{Hg}$. Occlusion of the aorta was confirmed with an arterial blood pressure transducer. A distal arterial port transducer also confirmed the SBP to be decreasing and dampened. The patient received additional 2 units of $\mathrm{pRBCs}$ and 2 units of FFP while on the way to the operating room.

The patient was then taken to the operating room for preperitoneal packing of the pelvis. The preperitoneal space was entered and a large hematoma was encountered. Seven kaolin-impregnated hemostatic gauze pads (QuikClot, Z-Medica, Wallingford, CT) were packed into the pelvis and the hemorrhage was noted to subside with no expansion of the hematoma. Once hemostasis was achieved, the aortic balloon was then deflated and no additional hemorrhage was seen for 35 $\min$.

There was blood-tinged fluid seen in the exposed peritoneum, so the midline incision was extended superior for an

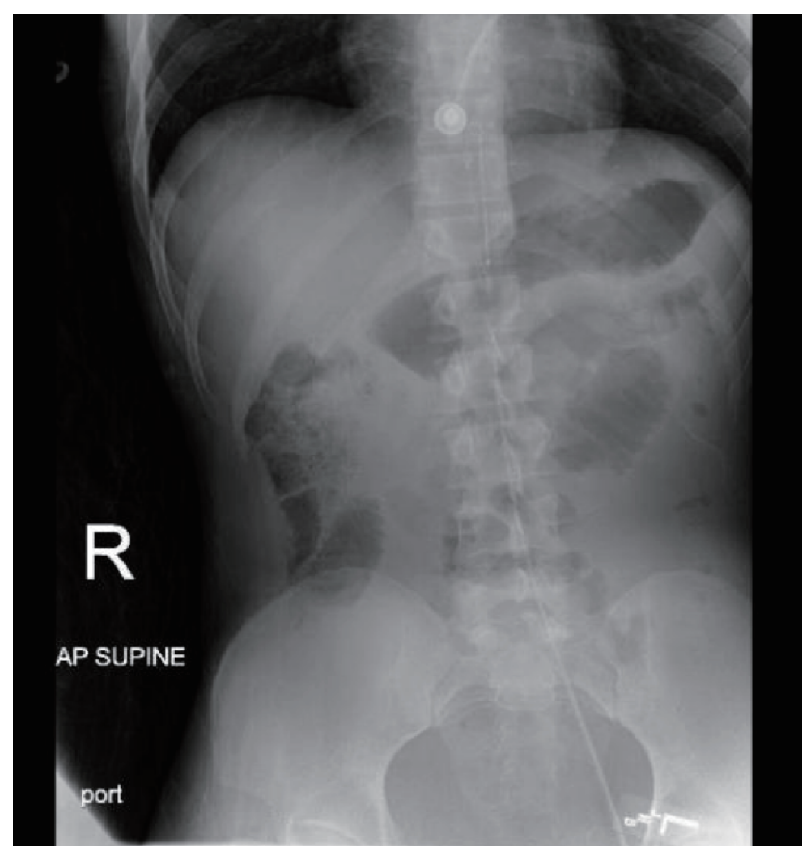

Figure 2. Abdominal X-ray confirming catheter tip placement in the infrarenal aorta, zone III.

exploratory laparotomy. Only $100 \mathrm{~mL}$ of blood was evacuated from the peritoneal cavity. All quadrants of the abdominal cavity were explored and found no injuries except a distended bladder with surrounding hematoma. A methylene blue dye test was performed via a clamped foley catheter. There was no extravasation of dye into the peritoneal cavity. The abdominal fascia was closed with the packings left in the preperitoneal space and a vacuum suction dressing put over the wound.

After the operation, the patient was immediately taken to the CT scanner for further evaluation of the abdomen and pelvis. The CT angiogram showed no definite active arterial extravasation of contrast. The CT cystogram (Fig. 3) however, showed contrast throughout the area of the previous pelvic hematoma, extending into the right inguinal region. There was an increase in extraperitoneal contrast after the cystogram suggesting bladder rupture with extravasation.

From the CT scanner, the patient was sent to the surgical intensive care unit (SICU) for further resuscitation. Vital signs upon arrival to the SICU were temperature $35.3{ }^{\circ} \mathrm{C}$, pulse 84 beats/min, blood pressure $80 / 60 \mathrm{~mm} \mathrm{Hg}$, respiratory rate of 15 breaths/min and oxygen saturation of $100 \%$ on $40 \%$ $\mathrm{FiO}_{2}$. In the SICU, the REBOA sheath was removed without any issues. Massive transfusion protocol (MTP) was, however, continued due to the persistent hypotension. Thromboelastography (TEG) was performed to guide the transfusion of blood products and 2 units of fresh frozen plasma (FFP) and 2 units of platelets were given. The patient continued to have intermittent episodes of hypotension and was interventional radiology was consulted. Bilateral angiogram of the internal iliac arteries was performed (Fig. 4). The right internal iliac artery showed a subtle blush suggestive of distal small vessel injury and the branches of the right internal iliac were gel-foam embolized. No other injuries or significant acute bleeding were found. 


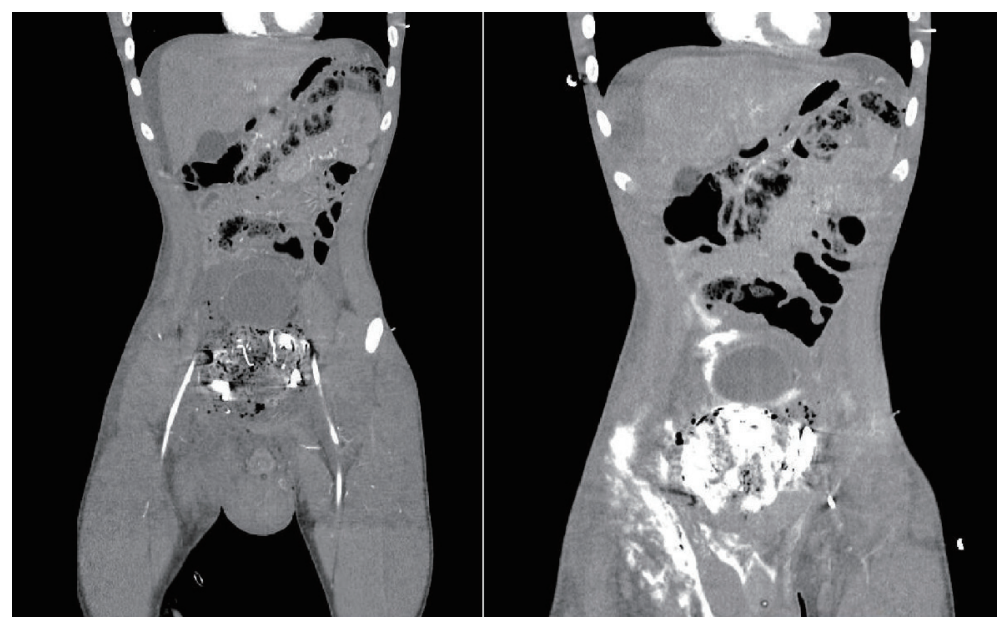

Figure 3. CT abdomen/pelvis with cystogram showing contrast throughout pelvic area and extending into the right inguinal region.

Blood pressures remained stable over the next several hours, but the patient was noted to have mottling of the right lower extremity with loss of pulses, along with no urine output. He was taken to the operating room again for removal of the preperitoneal packing, re-evaluation of the pelvic hematoma, and operative evaluation by urology and vascular surgery.

In the operating room, the kaolin-impregnated hemostatic gauze pads were removed from the preperitoneal space. Three sharp, jagged bone fragments were found in the right pelvis from the multiple pelvic fractures. There was venous bleeding in the area so a combination of oxidised regenerated cellulose absorbable hemostat gauze and sheets (Surgicel Nu-Knit and Surgicel SNoW, respectively; Ethicon, US. Cincinnati, $\mathrm{OH}$ ), as well as the flowable gelatin matrix with thrombin (Surgiflo, Ethicon, US. Cincinnati, OH) and laparotomy pads were used to obtain hemostasis. No other injuries were found in the preperitoneal space. The abdomen was then explored and no blood or injury was found except for a small serosal tear at the distal rectum, which was repaired.

Urology evaluated the bladder by removing the foley catheter and performing a flexible cystoscopy. A completely disrupted membranous urethra was found. Given the severity of his injuries, a vesicostomy with a 22Fr suprapubic catheter placement was performed. Vascular surgery then performed a right lower extremity angiogram via the right external iliac and found no injuries. The abdomen was left open with a pelvic drain and abdominal wound VAC dressing. The patient was then sent to the SICU for further resuscitation.

The next day, the patient was taken to the operating room. Extraperitoneal packing and laparotomy pads were removed and there was no evidence of further bleeding. A drain was placed and abdominal wound was closed. Orthopedic surgery immediately followed and performed a closed reduction and screw fixation of the pubic rami, sacroiliac joints, and left acetabulum.

On hospital day 5, the patient was taken to the operating room again for an open reduction internal fixation of the left acetabulum, and removal of left distal femoral traction pin. The patient remained stable and the remainder of his hospital course was uneventful. He was discharged to an acute rehabilitation facility on postoperative day 8 .

\section{Discussion}

Globally, trauma remains the leading cause of morbidity and mortality for all age groups with uncontrolled hemorrhage as the most common form of preventable death in the trauma setting. REBOA is a method of endovascular hemorrhage control that has existed since the Korean War in 1950 [2]. REBOA

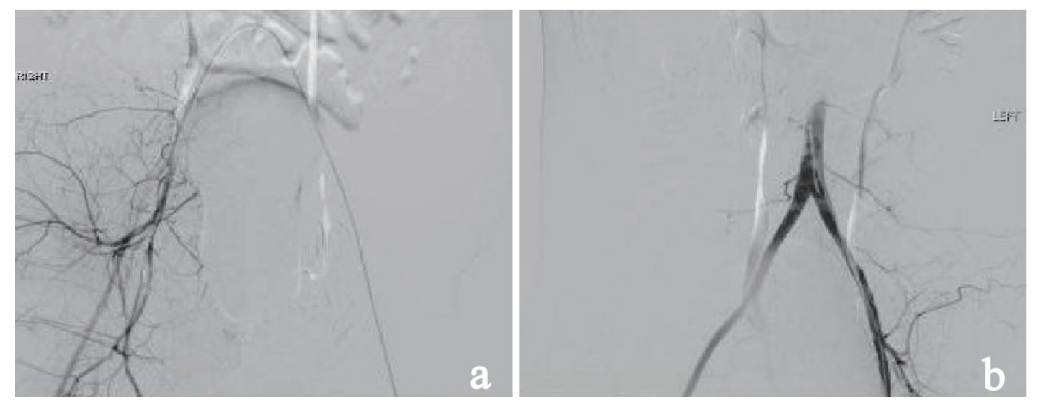

Figure 4. (a) Angiogram showing gelfoam embolization of the branches of right internal iliac artery. (b) Angiogram showing the right common iliac artery and right internal iliac artery with a subtle blush suggesting distal small vessel injury. 
involves the insertion of a balloon into the aorta via a femoral access approach that is subsequently inflated to provide hemostasis.

The procedure is best described as a resuscitation technique, which involves placing and inflating a balloon within the aorta proximal to the site of non-compressible hemorrhage. The current hospital standard for REBOA time of placement is approximately $6 \mathrm{~min}$ [3]. The goal is to support central pressure and restore hemostasis while minimizing bleeding, allowing perfusion to the brain and heart [4]. It is indicated in life-threatening hemorrhagic shock; most commonly used in the trauma setting, but has even been used in postpartum hemorrhagic shock [5]. There are five integral steps to performing the procedure [6].

The first step involves obtaining arterial access into the femoral artery. This can be achieved by open exposure of the femoral artery and external iliac artery. An 18 gauge hollow needle is inserted into the artery and a guidewire placed through the needle. Next, the needle is removed and an introducer sheath is inserted over the guidewire using the Seldinger technique to provide a functional port. The introducer sheath must be 12 Fr or larger. Step two involves selecting and positioning of the appropriate balloon. Balloons must be soft, complaint and of large diameter to permit successful occlusion of the aorta. Inflating the balloon in zone III provides occlusion for injuries specific to the pelvis or femoral hemorrhages. Step three is inflation of the balloon with saline according to the manufacturer specifications. It is important to take caution during this step and inflate the balloon slowly as rapid inflation of the balloon may result in circulatory collapse. Once effective inflation is achieved it is paramount to secure the balloon's position in order to maintain occlusion and ultimately maintain central pressure. Step four is deflation of the balloon. Similar to step three, it must be done with extreme caution and in the appropriate setting as deflation of the balloon carries the inherent risk of release of metabolic byproducts and resulting acidosis. Deflation should be achieved in a definitive care setting such as the operating room with the presence of anesthesia and the acute care surgeon. Step five is removal of the sheath, catheter and deflated balloon after hemorrhage is definitively controlled.

In our patient, the use of REBOA stabilized him until definitive operative repair could be undertaken. Once hemostasis was achieved the abdomen and pelvis were explored for further injuries. Although there was leakage of methylene blue when injected through the foley catheter, there was no intraperitoneal bladder injury identified. As this was not a life-threatening injury, resuscitation took priority and control of hemorrhage with bilateral hypogastric artery embolization was performed prior to repair of the urologic injury.

Prophylactic embolization of the hypogastric arteries is a common intervention for pelvic injury, particularly in hemodynamically unstable patients. In the face of massive pelvic fracture the first step is external pelvic fixation using towels, sheets, or a pelvic stabilization device or even operative placement of pins. Pelvic fixation can successfully arrest venous and smaller arterial bleeding as well as bleeding from bony surfaces. As was done in our case, embolization should be performed early in the control of arterial bleeding before severe coagulopathy develops [7].

Several hours after embolization of the branches of the right internal iliac artery, the patient developed mottling of the right lower extremity with loss of pulses. The angiogram performed during the embolization procedure did not show any injuries or acute bleeding of the vessels in either lower extremity. It was thought that the preperitoneal packing was causing excessive compression resulting in decreased pulses and urinary retention. This prompted the patient to be taken back into the operating room.

Limb ischemia is a known complication of REBOA, especially with larger sheath sizes such as the $12 \mathrm{Fr}$. A few institutional studies have shown the rate of limb ischemia can range from $0 \%$ to $20 \%$ depending on the sheath size used [8, 9]. The use of a 7Fr sheath, as with our patient, showed the lowest rate of limb ischemia, with some studies showing no complications at all [8-10]. For patients with limb ischemia, treatment included operative vascular surgical repair or amputation. As this is not a widely used procedure, further studies will be needed to determine the rate of limb ischemia and other complications.

While this technique has the potential to reduce the need for resuscitative thoracotomy, commonly used devices require large introducer sheaths (12Fr or greater) risking significant vascular injury and requiring formal surgical repair of the femoral artery upon removal. Furthermore, the requirement for long guidewires makes the procedure cumbersome anywhere other than an angiography suite. It is likely that the requirement for placing such large sheaths under emergency circumstances in addition to the need for cumbersome guidewires has inhibited the wide adoption of this technique. However, as in our case, recently approved devices designed to deploy through smaller 7Fr sheaths without the need for cumbersome guidewires have become available [11, 12].

In conclusion, this particular case is distinctive and unique in that a multidisciplinary approach was utilized in conjunction with a non-routine and minimally invasive method of control of hemorrhagic shock as well as the traditional laparotomy in trauma in order to control a life-threatening situation. This case also highlights the necessity for a multidisciplinary approach between the emergency physician, trauma surgeon, interventional radiologist, critical care physician, vascular surgeon and urologist. REBOA is a proven life-saving measure in the setting of acute non-compressible hemorrhagic shock. Despite its potential to save lives, non-vascular surgeons and other practitioners who care for patients in hemorrhagic shock may not consider the use of REBOA due to lack of knowledge, skill or equipment required to perform the procedure [13]. As a novel procedure, we believe it is being underutilized. This case study demonstrates the successful rescue of a patient with multiple severe injuries and hemorrhagic shock in which REBOA use was critical. This novel procedure allowed us to quickly stabilize the patient hemodynamically in order to address his life-threatening injuries. It has the unique function of being able to effectively control difficult life-threatening bleeds without undergoing extensively invasive procedures and without a large thoracotomy incision. REBOA has a definite role with a vast potential to save a crashing patient. It can significantly add to a provider's 
armamentarium in the trauma patient.

\section{Conflict of Interest}

There is no disclosure or conflict of interest in this project.

\section{References}

1. Manzano Nunez R, Naranjo MP, Foianini E, Ferrada P, Rincon E, Garcia-Perdomo HA, Burbano P, et al. A metaanalysis of resuscitative endovascular balloon occlusion of the aorta (REBOA) or open aortic cross-clamping by resuscitative thoracotomy in non-compressible torso hemorrhage patients. World J Emerg Surg. 2017;12:30.

2. Moore LJ, Brenner M, Kozar RA, Pasley J, Wade CE, Baraniuk MS, Scalea T, et al. Implementation of resuscitative endovascular balloon occlusion of the aorta as an alternative to resuscitative thoracotomy for noncompressible truncal hemorrhage. J Trauma Acute Care Surg. 2015;79(4):523-530; discussion 530-522.

3. Ross EM, Redman TT. Feasibility and proposed training pathway for austere application of resuscitative balloon occlusion of the aorta. J Spec Oper Med.18(1):37-43.

4. Valkenburg A, Bennett D, Bishop J, Smith G. Resuscitative endovascular balloon occlusion of the aorta as a potential prehospital procedure for the control of non-compressible haemorrhage: A literature review. Australasian Journal of Paramedicine. 2015;12(4).

5. White JM, Cannon JW, Stannard A, Burkhardt GE, Spencer JR, Williams K, Oh JS, et al. Direct vascular control results in less physiologic derangement than proximal aortic clamping in a porcine model of non- compressible extrathoracic torso hemorrhage. J Trauma. 2011;71(5):1278-1286; discussion 1286-1277.

6. Stensaeth KH, Sovik E, Haig IN, Skomedal E, Jorgensen A. Fluoroscopy-free Resuscitative Endovascular Balloon Occlusion of the Aorta (REBOA) for controlling life threatening postpartum hemorrhage. PLoS One. 2017;12(3):e0174520.

7. Stannard A, Eliason JL, Rasmussen TE. Resuscitative endovascular balloon occlusion of the aorta (REBOA) as an adjunct for hemorrhagic shock. J Trauma. 2011;71(6):1869-1872.

8. Bogert JN, Davis KM, Kopelman TR, Vail SJ, Pieri PG, Matthews MR. Resuscitative endovascular balloon occlusion of the aorta with a low profile, wire free device: A game changer? Trauma Case Rep. 2017;7:11-14

9. Taylor JR, 3rd, Harvin JA, Martin C, Holcomb JB, Moore LJ. Vascular complications from resuscitative endovascular balloon occlusion of the aorta: Life over limb? J Trauma Acute Care Surg. 2017;83(1 Suppl 1):S120-S123.

10. Saito N, Matsumoto H, Yagi T, Hara Y, Hayashida K, Motomura T, Mashiko K, et al. Evaluation of the safety and feasibility of resuscitative endovascular balloon occlusion of the aorta. J Trauma Acute Care Surg. 2015;78(5):897-903; discussion 904.

11. Lopera JE. Embolization in trauma: principles and techniques. Semin Intervent Radiol. 2010;27(1):14-28.

12. Belenkiy SM, Batchinsky AI, Rasmussen TE, Cancio LC. Resuscitative endovascular balloon occlusion of the aorta for hemorrhage control: Past, present, and future. J Trauma Acute Care Surg. 2015;79(4 Suppl 2):S236-242.

13. Teeter WA, Matsumoto J, Idoguchi K, Kon Y, Orita T, Funabiki T, Brenner ML, et al. Smaller introducer sheaths for REBOA may be associated with fewer complications. J Trauma Acute Care Surg. 2016;81(6):1039-1045. 